\title{
Performance analysis of wireless sensor network with load balancing for data transmission using xbee $\mathrm{zb}$ module
}

\author{
Ahmad Yusuf Ardiansyah ${ }^{1}$, Riyanarto Sarno ${ }^{2}$ \\ ${ }^{1}$ Department of Information Technology Management (MMT), Institut Teknologi Sepuluh Nopember (ITS), Indonesia \\ ${ }^{2}$ Department of Informatics, Institut Teknologi Sepuluh Nopember, Indonesia
}

\begin{tabular}{l} 
Article Info \\
\hline Article history: \\
Received Jul 30, 2 \\
Revised Sep 11, 20 \\
Accepted Oct 4, 2019 \\
\hline Keywords: \\
Data transmission \\
Load balancing \\
Mesh topology \\
Non-balancing \\
Qos \\
Routing \\
Zigbee
\end{tabular}

\section{INTRODUCTION}

The wireless sensor network (WSN) is a sensor network infrastructure that is connected wirelessly. Increasingly rapid technological developments have led to the emergence of various needs in fields such as environmental monitoring, smart metering, logistics, smart agriculture, one of the part in industry 4.0 system exactly for connecting system, and industrial control. In the near future, home, smart cities, and industrial sectors will implement complex wireless sensor networks to their accessibility through the internet network [1].

Although there are many advantages of WSN, it still has a limitation in the aspect of energy, limited storage capacity, unreliable routing and lack of supervision on the distribution that can affect the quality of the informed data [2]. Moreover, the efficiency of routing protocols in WSN plays an essential role in the transmission of the data packet.

\begin{abstract}
In general, research in the field of wireless sensor network (WSN) has never discussed the reliability aspect of network routing with router devices that for damage occurs. To date, overloaded routers will be of network performance. Therefore, we propose research using the AODV routing, and Mesh routing algorithm to find other routes as an alternative when problems occur and using the Round Robbin based xbee algorithm on providing load balance control carried out by the router. The experiments on the performance of non-balancing networks and balancing were conducted. Both trials used quality of service (QoS) parameters as a guarantee of performance to be more effective and in line with expectations. Measurements performed by testing the parameters of packet loss, delay, throughput, and fault tolerance. The network performance in finding other alternative routes has been successfully carried out by transmitting 100 packets from the end device node to the coordinator node via the router based on distance variations from 0 to 100 meters. The recovery time required by the dead router to find another route was 10 seconds, this was related to the parameter delay, and fault tolerance. The experimental results of the nonbalancing system showed an average $20 \%$ packet loss in one transmission, meanwhile the packet loss was smaller than the previous experiment by $37 \%$. that could improve QoS performance by $17 \%$.
\end{abstract}

Copyright $(0) 2020$ Institute of Advanced Engineering and Science. All rights reserved.

\section{Corresponding Author:}

Riyanarto Sarno,

Institut Teknologi Sepuluh Nopember,

Jalan Raya ITS, Keputih, Sukolilo, Kota Surabaya, Jawa Timur 6011, Indonesia.

Email: riyanarto@if.its.ac.id 
Routing protocols play an important role in developing the WSN network with high service quality. By setting up sophisticated routing protocols can reduce the limited resources possessed by the WSN devices used. In telecommunication and computer science, wireless sensor network is the active area of research. It plays a major role in many areas of research [3]. All applications are involved by WSN such as health care monitoring [4], area monitoring [5], quality management [6], air pollution monitoring [7], agricultural management [8], nature disaster prevention [9], electrical nose [10] and data logging have used high traffic for distributing the essential data can reduce the quality of data reliable [11]. One example of an event which can affect the quality of data transmission is "Bottleneck" and over workload. Bottleneck is a traffic jam due to excessive load, while over workload is a term of excessive workload. In this survey load balancing issue is considered. Load balancing typically is not equally distributing load on all nodes in the network, it involves identifying the optimal load on nodes of the network to increase its lifetime [12].

Various studies related researches the routing method of sensor networks attempted to reduce all network loads to extend and break down traffic with various approaches [4-9]. Previous research also tried to apply simulate various WSN topologies by measuring QoS [13, 14]. In addition. However, the reduction of lifetime, limited storage capacity and traffic is inevitable in the static and in the protocol network of Zigbee. The monitoring, and balancing of the information is very essential. So further research is needed to design a system that can help to monitoring, controlling, and balancing of transmission data in the field of routing in Zigbee.

Therefore, we propose the renewal of dynamic routing and load balancing in WSN using the AODV routing [15], Mesh routing algorithm to find alternative routes when problems occur $[1,6,7,9,16]$ and using the Round Robbin for Zigbee scheduling algorithm for balancing data transmission. A system and method is proposed for all applications are involved by WSN such as health care monitoring, area monitoring, quality management, air pollution monitoring, agricultural management, nature disaster prevention, etc. We have also tested the hardware reliability and network performance measurements in outdoor spaces where the results have proven effective in increasing the reliability and effectiveness of network performance.

\section{THE PROPOSED ZIGBEE ARCHITECTURE COMMUNICATION}

For the proposed model, we use the AODV routing algorithm, and Mesh routing to look for other alternative routes when problems occur, to provide control of the load balance that is run on the router Zigbee protocol. The following is the theory presented.

\subsection{Wireless Sensor Network (WSN) Application}

Wireless communication design describes the parts and supporting aspects in building wireless media communication, in this case the Zigbee protocol. In designing wireless communication, there are basics that must be done in managing the desired communication. In addition, the proposed methodology also helps ensure all the aspects run well. Wireless Mesh network is a network in which each node can communicate with many other nodes. Wireless sensor network (WSN) is a wireless network that uses sensors to monitor physical or environmental conditions. Wireless sensor is usually used for monitoring functions to measure physical quantity, such as temperature, pressure, and humidity, and to send the data to data concentrator.

\subsection{Zigbee Protocol}

Zigbee is IEEE 802.15.4 MAC and layer PHY protocol designed for a minimalist scale including low bandwidth, low cost, low power, and wireless network standard [1]. Mesh network provides high reliability and long distance coverage with low power and long durability. It is usually applied to systems that implement monitoring and controlling on wireless system applications.

\subsection{Zigbee Data Transmission}

The important role of data transmission into all Zigbee data packages are sent using wireless hardware and application layer addressing field [17]. Zigbee data package can be sent as unicast or broadcast shipping modes.

\subsubsection{Unicast and Broadcast}

Unicast Figure 1 transmission is sending data from one device to another destination source device. Unicast is the only mode that supports repetition. In this mode, the receiver module sends an RF acknowledgment (ACK) package to the transmitter. If the sender module does not accept ACK, the package will be resent three times or until the ACK is received. The following is an example image for the xbee device setting that sends data between the router and coordinator. 
Meanwhile, the broadcast transmission Figure 2 of all nodes in a network can receive broadcast data sent. For broadcast mode, one of the nodes will read the information, the destination low (DL) value is changed to "OxFFFF" and the destination high (DH) value becomes "0". Whereas on the other nodes, the DL is filled with SL from the node that broadcasts the information.

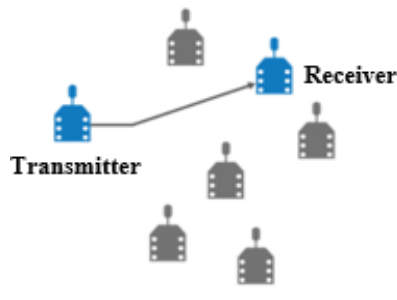

Figure 1. Unicast mode

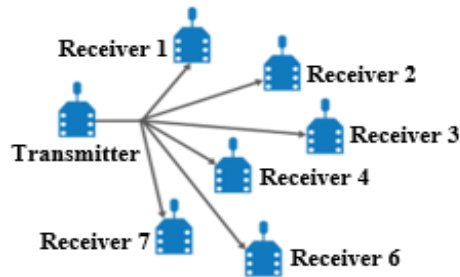

Figure 2. Broadcast mode

\section{RESEARCH METHOD}

The main objective of this research was to implement the renewal of the wireless sensor network system by building a dynamic routing system that could perform balancing when overloaded. This section will discuss the literature review or the theoretical basis used in completing this study. The method used in this study was an experimental method that applied AODV Mesh Routing and load balancing algorithms to support the reliability of wireless data transmission.

\subsection{Hardware Architecture}

This wireless sensor network was designed using five xbee modules, five arduino, five xbee USB boards, and three DHT22 sensors. XBee configuration was carried out using the XCTU framework. This setting was conducted to determine the xbee device as an end device, router and xbee coordinator. We used DHT22 type as the temperature sensor, it was connected to the end device to measure the temperature and humidity around it [18]. Table 1 shows the tools used in this study.

Table 1. Hardware Used

\begin{tabular}{cl}
\hline Hardware & \multicolumn{1}{c}{ Function } \\
\hline XBee/XBee-PRO S2C 802.15.4 & XBee end device, XBee router, XBee coordinator \\
Arduino Uno & As a microcontroller of serial communication and data process \\
USB Explorer Board All & As a device to set the XBee S2C configuration \\
DHT22 & As a temperature sensor device \\
\hline
\end{tabular}

Table 1 shows the list of hardware used in this study. The setting of xbee network configuration used XCTU firmware, XCTU firmware is the official configuration program for xbee devices provided by Digi Inc. The configuration type of the selected device was the representative of the vital role in wireless data transmission. Some of these roles were xbee as coordinator, two xbee modules as routers, and three xbee modules as end devices [19]. The arduino board used as a microcontroller that processed xbee address storage, declarations of data and variable length, prepared data transmission schedules between xbee, and carried out routing and balancing functions using serial communication. Supporting the communication process between xbee and the arduino microcontroller, a USB Explorer Board [20] needed which was installed into one section with the xbee module to facilitate the process of wiring pins in and pinouts. All xbee DHT22 modules were a sensor that worked by detecting temperature and humidity. This sensor was connected to arduino via serial communication and used digital data types [21, 22].

Figure 3 is an illustration of the hardware architecture that has implemented in this study. The architecture was divided into three parts of processes, the first process was detecting, the second process was routing and balancing, and the third process was performance measurement. Figure 3 shows the input process using three end devices as wireless media that directly connected to the arduino and DHT22 sensors. Each input data received by arduino (microcontroller) was delivered using serial communication [23]. The length of data in a single transmission has determined and limited. The length of the data size [leghtdatastore] has been determined for each end device. The number of characters of the data used was 50 bytes because the xbee device could only send data packets in a single transmission of 256 bytes. Thus it was necessary to make a schedule of data transmission between xbee devices [24]. 


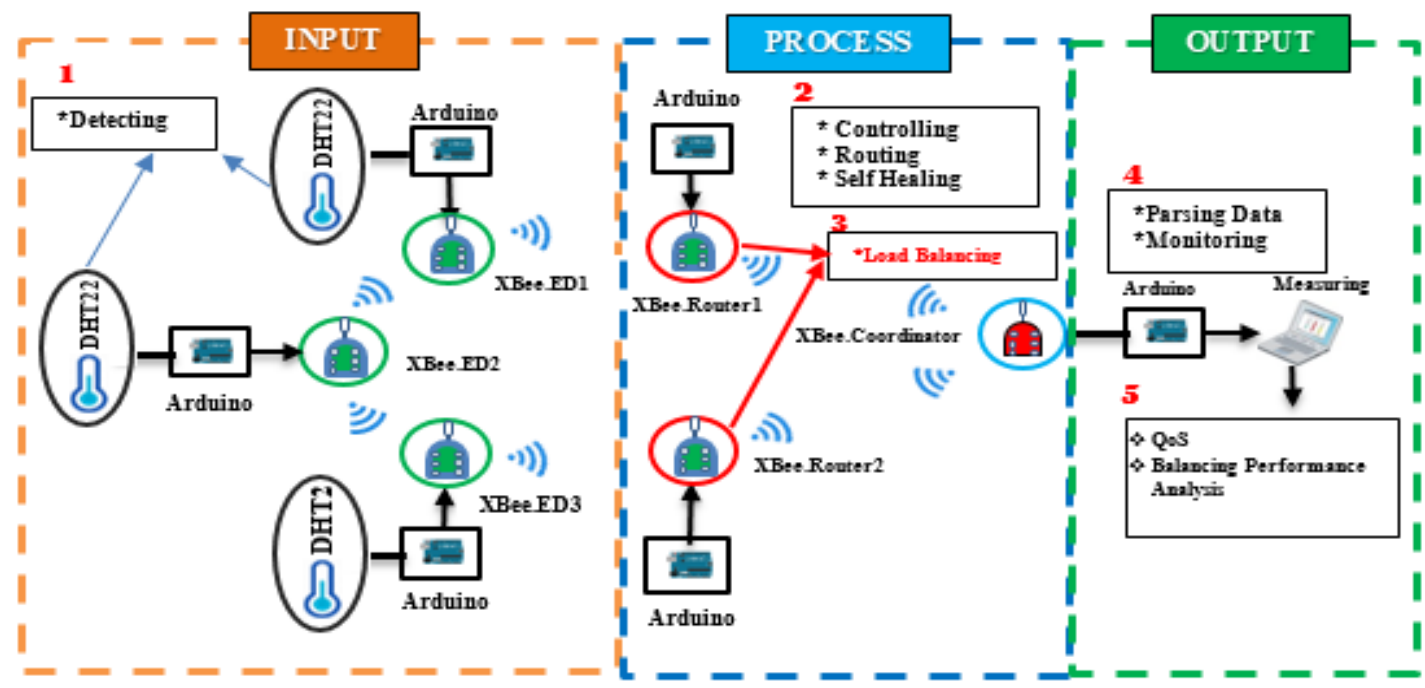

Figure 3. Hardware architecture

Two xbee modules were used as routers to control system balancing, it represented the function of the router as a full or (FFD) function device [25]. Therefore, we carried out three process scenarios on router devices, namely routing, self-healing, and load balancing. The final process was output, where WSN performance was measured by using QoS parameters consisting of throughput, packet loss, delay, and fault tolerance [26].

\subsection{Zigbee Mesh Routing Protocol}

Ad hoc On-Distance Vector (AODV) is one of the routing protocol designed for ad hoc wireless networks that are categorized as a reactive routing protocol. AODV has main components such as sequence numbers, ID numbers, RREP, RREQ, and data messages. These components were very supportive during the route path (discovery) and route maintenance process. Illustrations of the application of route path (discovery) and route maintenance shown in Figure 4 and 5.

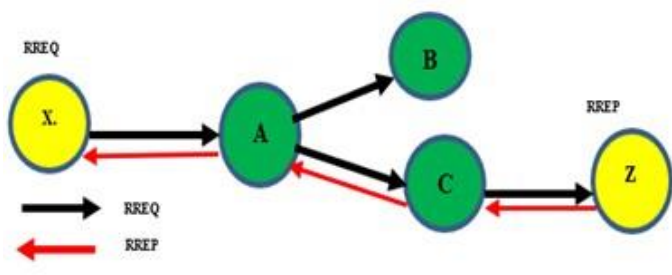

Figure 4. Route discovery

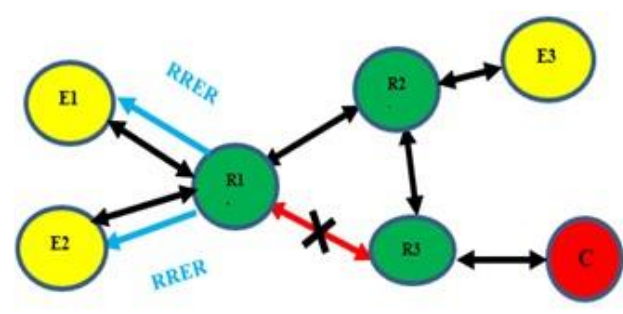

Figure 5. Route maintenance

\subsection{Route Discovery}

Route discovery is a condition of the system finding new route information. To perform route discovery, the origin node broadcasted a request (RREQ) to the nearest neighbor to find out the sequence number of the destination ID.

Figure 4 shows the route discovery using the AODV method, where the $\mathrm{X}$ sign as the source node and the $\mathrm{Z}$ sign as the destination node. When the destination route received a request from the primary node, the node checked the ID number for re-verification to ensure that the packet was valid. Then, the destination node replied to the RREQ packet with the route replay packet (RREP).

\subsection{Route Maintenance}

Route maintenance is the condition of the system to repair damaged routes. This problem usually occurs if a link to the next destination node cannot be detected by using the route discovery method, then the 
link will send a route error message (RRER) to its neighbor node until it spread to the source node. Figure 4 shows the steps of the route maintenance in Zigbee routing.

Figure 5 is an illustration of a route maintenance scheme using the AODV method, where the X sign as the source node and the $\mathrm{Z}$ node sign as the destination node. When the node could not forward the data packet, then the node would send a route error message to the address of the source that was E1 and E2. Then, the checking and confirmation of the new route were performed against the new route of R2. If node E1 and E2 have verified the new node address, then the system will delete route R3, and the packet can send via the new route.

\subsection{Load Balancing Implementation on Zigbee Protocol}

The technique used in implementing load balancing systems was the Round Robbin technique [24]. This algorithm divided the load in rotation and sequentially from one router to another so that a round could be formed. In simple terms, the balancing system will work if the storage on the router 1 has received temporarily 10 data packets on one shipment. Furthermore, router 1 requested the end device to perform the switching address (SA) to router 2. The purpose of this SA was to switch the route that the end device will pass from router 1 to router 2 . The balancing system would automatically divide the load based on the maximum number of packets received. The number of packet loads specified on the router was 10 packets with a maximum character of 2000 bytes because those packets could be sent at one time between xbee routers and microcontroller arduino.

\subsection{Data Transmission Scenario}

Packet transmission without balancing applied a self-healing technique from the Zigbee Mesh Routing protocol, which both routers serve as a liaison and control in managing the transmission route [25]. The first assumed scenario was router 1 has an error. Thus the transmission of data packets must be routed to router 2 to continue the transmission from router 1 . The routing system would respond by searching for other routes that could be passed.

Figure 6 shows that end device 1, end device 2, end device 3 sent data packets through router 1, and 2. router 1 and 2 only received data packets from the end device during the transmission process without carrying out a load limit process. For router 1 to know the address of router 2, the transmission mode on both routers was set as a peer to peer and peer to multipoint communication or unicast and broadcast communication in the Zigbee network.

The second scenario was seen in Figure 7, the rule system used was to ensure that the two routers were active. The first thing that should be considered by the end device before transmitting a data packet was checking the status of the router. If the conditions of the two routers are active, the system balancing will respond and be ready to receive packets from the end device with a maximum specified load.

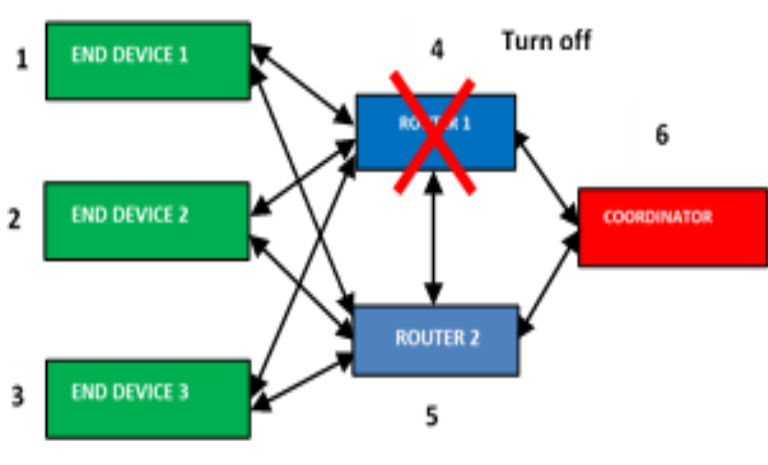

Figure 6. Non-balancing scheme

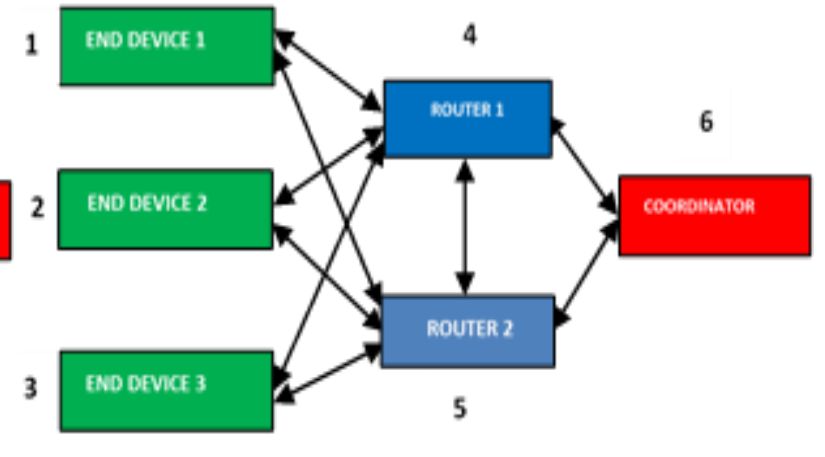

Figure 7. Balancing scheme

\subsection{Rule System Scenario}

This scenario system provided information about routing in a system by applying the balancing and non-balancing. Routing information that was conveyed through the tables below represented the conditions of the network testing of the died router (off) and the maximum condition of the packet load received. The maximum load received by each router did not exceed 100 packets. The rule system of non-balancing worked on a router that had a disturbance. Thus, it required to switch to another line. On the other hand, if the two routers are active, then the system balancing rule will be used. The rule system is shown in Table 2. 
Table 2. Non-Balancing Rule

\begin{tabular}{|c|c|c|c|c|}
\hline No & Rule & Routing & Route Status & Router Maintenance \\
\hline 1 & & Routing 1 & Router 2 Off & Source ED1,2,3 -> Router 1 -> Coordinator \\
\hline 1 & Non-Balancing & Routing 2 & Router 1 Off & Source ED1,2,3 -> Router 2 > Coordinator \\
\hline
\end{tabular}

Non-balancing is a load equalization technique without using a control system. Table 2 shows that there two routes that could be traversed by data packets namely routing 1 and routing 2 . In Routing 1 , all data packets were sent from the end device to the coordinator via router 1. In Routing 2, all data packets were sent from the end device to the coordinator via router 2 . Each routing required to turn off the router device so that non-balancing conditions could run effectively. This routing assumed to be like applying router maintenance on a Zigbee Mesh routing network.

Balancing is a technique of dividing loads in turns and sequentially from router 1 to router 2. Based on Table 3, two routers represented each routing. The balancing system will work if the number of packets received by router 1 has reached the maximum limit. The switching address function on the router served to respond to router 2 to forward data packets received from the router 1 . The maximum value required by router 1 and router 2 was 100 data packets. The illustration of the switching address on the router shown in Figure 8.

Table 3. Balancing Rule

\begin{tabular}{ccccc}
\hline No & Rule & Routing & Router Max Load & Router Maintenance \\
\hline \multirow{2}{*}{1} & \multirow{2}{*}{ Balancing } & Routing 1 & Router 1 = 100 Packets & Source ED1,2,3 -> Router 1 -> Coordinator \\
& Routing 2 & Router 2 = 100 Packets & Source ED1,2,3 -> Router 2 > Coordinator \\
\hline
\end{tabular}

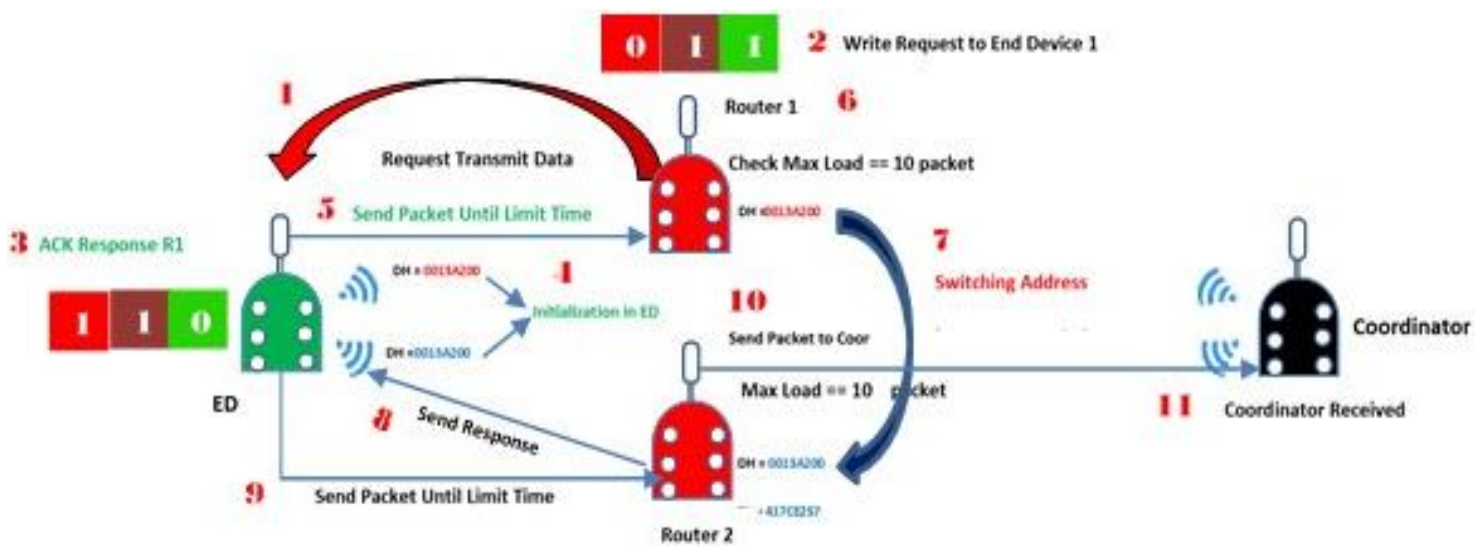

Figure 8. Switching address process

Based on Figure 8 in simple illustration, the switching address process is the process of replacing the address of router 1 to the address of router 2 . The switching address process located in the eleventh step. This step can be fulfilled if router 1 has reached the maximum packet received in one transmission. The condition of the load limitation given to the router aims to regulate the communication between the end device and the coordinator and regulate the memory capacity on arduino. The ability of the router as network control can be relied upon in finding alternative routes when problems occur. Therefore, this process is called dynamic routing that supports the reliability aspects of the WSN. Following are the reasons for using the max load on the packet router:

a) Required of balancing and routing system

b) Payload packet data

c) Total used flash memory

d) Available flash memory (SRAM) in microcontroller Arduino

$$
\begin{aligned}
& =1295 \text { bytes } \\
& =500 \text { bytes } \\
& =1795 \text { bytes } \\
& =205 \text { bytes or } 10.5 \%
\end{aligned}
$$

\subsection{QoS (Quality of Service)}

QoS or quality of service is a mechanism that allows network applications or services to operate as expected. Service quality guarantees network performance. In ensuring superior network performance, network parameters were needed to be able to evaluate each requirement required in the scenario, including 
quality of service, delay tolerance (delay), loss tolerance (packet loss), capacity (throughput), and fault tolerance [20].

\subsection{Throughput}

It shows the amount of packet data received at the destination node compared to the travel time written in units of bits per second (bps).

$$
\text { Throughput }=\frac{\sum \frac{i=T_{t+1}}{i=T} P_{i}}{T} ; 0 \leq t \leq T
$$

$\sum \mathrm{Pi}$ is the number of packets received in units (bits), and $\mathrm{T}$ is the total transmission time. The whole transmission time can be determined by finding the difference between the time of receipt and the time of transmission. The total number of packets that were successfully sent to the destination node is divided by the duration of the delivery time interval. Tt is the duration of the package delivery process that always increases during the transmitting process. Using of throughput measurements can produce information about effective data transfer rates measured in bps.

\subsection{Packet Loss Ratio (PLR)}

The packet loss is a parameter that describes a total number of lost packets. The packet failure reaches its destination, can be caused by several possibilities, such as congestion, limited memory, and media.

$$
\text { Packet Loss Ratio }=\left(\frac{\sum \frac{i=T_{t+1}}{i=T} D_{i}}{\sum \frac{i=T_{t+1}}{i=T} S_{i}}\right) x 100 ; 0 \leq t \leq T
$$

$\sum \mathrm{Di}$ is the number of packets lost, $\sum \mathrm{Si}$ is the number of packets that were successfully sent, and $\mathrm{Tt}$ is the sampling time (s). To find out the number of packets lost in one transmission, the package needs to be multiplied by $100 \%$. This is the standard value of the packet loss. The formula for this packet loss is obtained from the calculation of the number of packages received, then divided by the number of packets received, and then multiplied by $100 \%$. From the measurement results which are obtained are matched with the quality standards for package loss [19].

\subsection{Delay}

Delay is the difference between the time the packet data is sent and the time the data is received is the packet. The delay in a network can be used as a reference in assessing the quality of data transmission. As a result of the delay, the data we receive will experience a delay in coming time so that it reaches its destination.

$$
\text { Delay }=\frac{\sum \frac{i=T_{t+1}}{i=T} R T_{i}-\sum \frac{i=T_{t+1}}{i=T} S T_{i}}{\sum \frac{i=T_{t+1}}{i=T} R P_{i}} ; 0 \leq t \leq T
$$

$\sum \mathrm{RTi}$ is the number of packets received in one time, $\sum \mathrm{STi}$ is the number of packets sent at one time, and $\sum \mathrm{RPi}$ is the number of packets received. To find out the difference between the packet transmission time and the packet receipt time, the three formulas are reduced and then divided by the number of packets received. Tt is the duration of the package delivery process that always increases during the transmitting process, and $\mathrm{Tt}$ is the sampling time (s).

\subsection{Fault Tolerance (Recovery Time for Router)}

Fault tolerance in WSN is the network's ability to tolerate errors that cause service failure. It was included as an aspect of network resilience. This service focused on measuring the time of node failure detection or observing recovery time when a route failure occurs. 


\section{RESULTS AND DISCUSSION}

This study analyzed the data transmission performance by applying two scenarios. Namely, the first scenario was to analyze the data transmission without implementing a load balancing system (non-balancing), and the second scenario was to analyze the data transmission by implementing a load balancing system. We compared the network performance before using balancing and after using balancing in this section. The final result in this study was a comparison of the performance analysis between dynamic routing and balancing proposed, against the results of previous studies.

\subsection{XBee Configuration}

XBee connected to the XCTU framework via the serial COM port settings to configure xbee. The following is the illustration result of scanning xbee devices through XCTU. The configuration of the three xbee modes conducted with several serial communication basic configuration parameters such as, baudrate: 9600 , parity: none, stop bits: 1, data bits: 8 and flow control: none. The next configuration was to set the network id address, network channel, source address (SH+SL), destination address (DH+DL). All these configuration parameters declared in the arduino microcontroller which was processed using based C-programming.

\subsection{Hardware Design}

The purpose of designing the hardware was to avoid and reduce noise caused by untidy cabling. The implementation of the hardware built is seen in Figure 9.
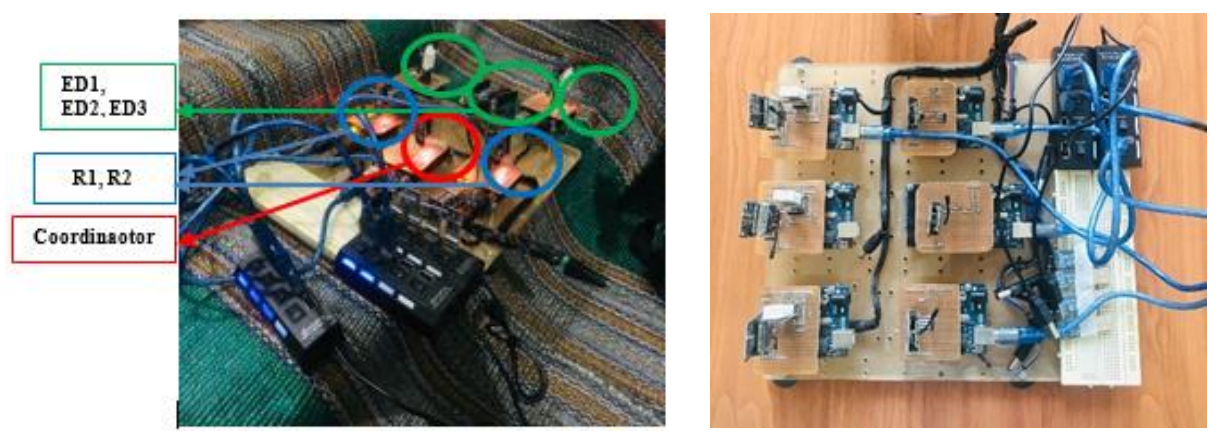

Figure 9. Hardware design

Acrylic with a thickness of $0.3 \mathrm{~mm}$ used as the base of the hardware board. Besides, the extended USB as an additional USB port, three modules of end devices, two routers, and one coordinator was also utilized to design the hardware. The $\mathrm{ABC} 9 \mathrm{~V}$ battery was used when performing a distance test. The electric circuit on this board was $9 \mathrm{~V}$ static which was installed in parallel.

\subsection{Arduino Test}

The experiments of the entire system in this study were processed on the arduino microcontroller. Data and variable length declarations, the function of processing data transmission schedules inter- xbee, as well as routing and balancing functions using serial communication. The following are the results of research experiments with arduino framework.

\subsection{The Test for Load Balancing System}

The experimental results of the self-healing and balancing tests prove the reliability and effective aspects in terms of data transmission in the WSN. This experiment has been successfully implemented using an arduino microcontroller, as shown in Figure 10. Load balancing system is a system designed to control workload. Excessive workload will reduce network performance on its storage media, so there needs to be a control mechanism on wireless devices. Based on the result of the trial balancing system in Figure 10, ED1, $\mathrm{ED} 2$, and ED2 receive a response from router 2 in the form of a "router full" meaning that the system balancing is active by limiting the receipt of the number of packages received by the router 2 . The number of initial packages predetermined for this study is a maximum of 10 packages in consideration of maintaining the stability of the router and the limitations of the specifications of the microcontroller device on the device router. 
Figure 10 shows the end device mechanism for transmitting and switching address data packets to the coordinator distinguished by transmission delay. ED1, ED2, and ED3 transmitted data packets every two seconds, three seconds, and five seconds, respectively to avoid the crash between the packets transmitted. The coordinator managed to receive data from ED1, ED2, and ED3 through routing 2 using serial application. Based on balancing system an experiment results able to transmit packet 87 out of 100 packets from end device to coordinator were transmitted approximately 9 minutes 35 seconds. In the delivery time, the system sends 100 packets via routing 1 or routing 2 . The packet transmission was more effective 2.20 minutes compared to the standard xbee transmission using the XCTU framework from Digi Inc.

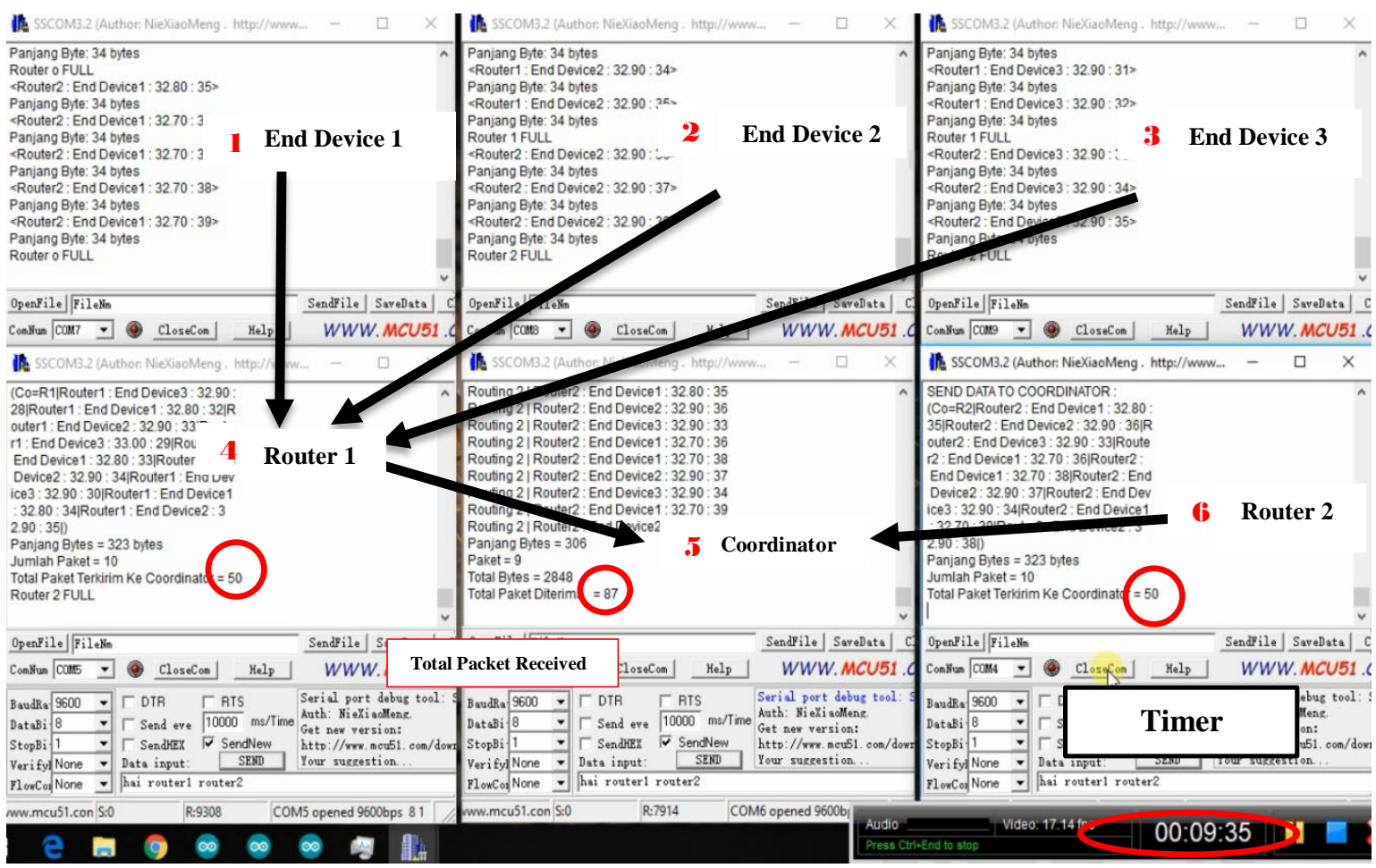

Figure 10. Balancing router

\subsection{Performance Analysis of QoS Parameter (Throughput, Packet Loss, Delay, and Recovery Time)}

The QoS performance test results provide supporting evidence to ensure superior network performance. The following are the performance parameters in quality of service, namely; delay, packet loss capacity, throughput, and recovery time.

Based on Table 4 the result of the trial and analysis of QoS are divided into two rule systems. The non-balancing rule produces an average value of packet loss of $37 \%$ with an average delay of 9.1 second, while the standard xbee throughput remains in the low category. The comparison of balancing measurement produces an average value of packet loss of $20 \%$, with an average delay of 6.8 seconds.

The expected criterion of this measurement is the greater throughput value represents the larger bandwidth allocation for transferring data. Throughput analysis shows that the average bandwidth allocation for the non-balancing is 46.9, and the average bandwidth for the balancing is 59.4. It means that of the two rule performances, the throughput of the non-balancing is smaller than the balancing because the packets transmitted in the rule balancing is controlled to minimize packet loss.

Based on Figure 11 the expected criterion of this measurement is the greater throughput value represents the larger bandwidth allocation for transferring data. Throughput analysis shows that the average bandwidth allocation for the non-balancing is 46.9, and the average bandwidth for the balancing is 59.4. It means that of the two rule performances, the throughput of the non-balancing is smaller than the balancing because the packets transmitted in the rule balancing is controlled to minimize packet loss. 
Table 4. Performance Analysis based on QoS Parameters

\begin{tabular}{|c|c|c|c|c|c|c|c|c|c|c|}
\hline No & Rule & Range & End Device & $\begin{array}{c}\text { Router } \\
1\end{array}$ & $\begin{array}{c}\text { Router } \\
2\end{array}$ & COOR & $\begin{array}{c}\text { Throughput } \\
\text { (KBps) }\end{array}$ & $\begin{array}{c}\text { PacketLos } \\
\text { s (\%) }\end{array}$ & $\begin{array}{l}\text { Delay } \\
\text { (second) }\end{array}$ & $\begin{array}{c}\text { Recovery } \\
\text { Time }\end{array}$ \\
\hline \multirow{2}{*}{1} & Balancing & 50 meter & 100 packet & 80 & 78 & 75 & 55.6 & $25 \%$ & 7.2 & $12 \mathrm{~s}$ \\
\hline & & 100 meter & 100 packet & 58 & 50 & 45 & 33.3 & $55 \%$ & 12.0 & $13 \mathrm{~s}$ \\
\hline \multirow[t]{3}{*}{2} & Balancing & 10 meter & 100 packet & 95 & 91 & 87 & 64.4 & $13 \%$ & 6.2 & $4 s$ \\
\hline & & 50 meter & 100 packet & 88 & 82 & 82 & 60.7 & $18 \%$ & 6.6 & $3 \mathrm{~s}$ \\
\hline & & 100 meter & 100 packet & 76 & 65 & 78 & 57.8 & $22 \%$ & 6.9 & $5 \mathrm{~s}$ \\
\hline
\end{tabular}

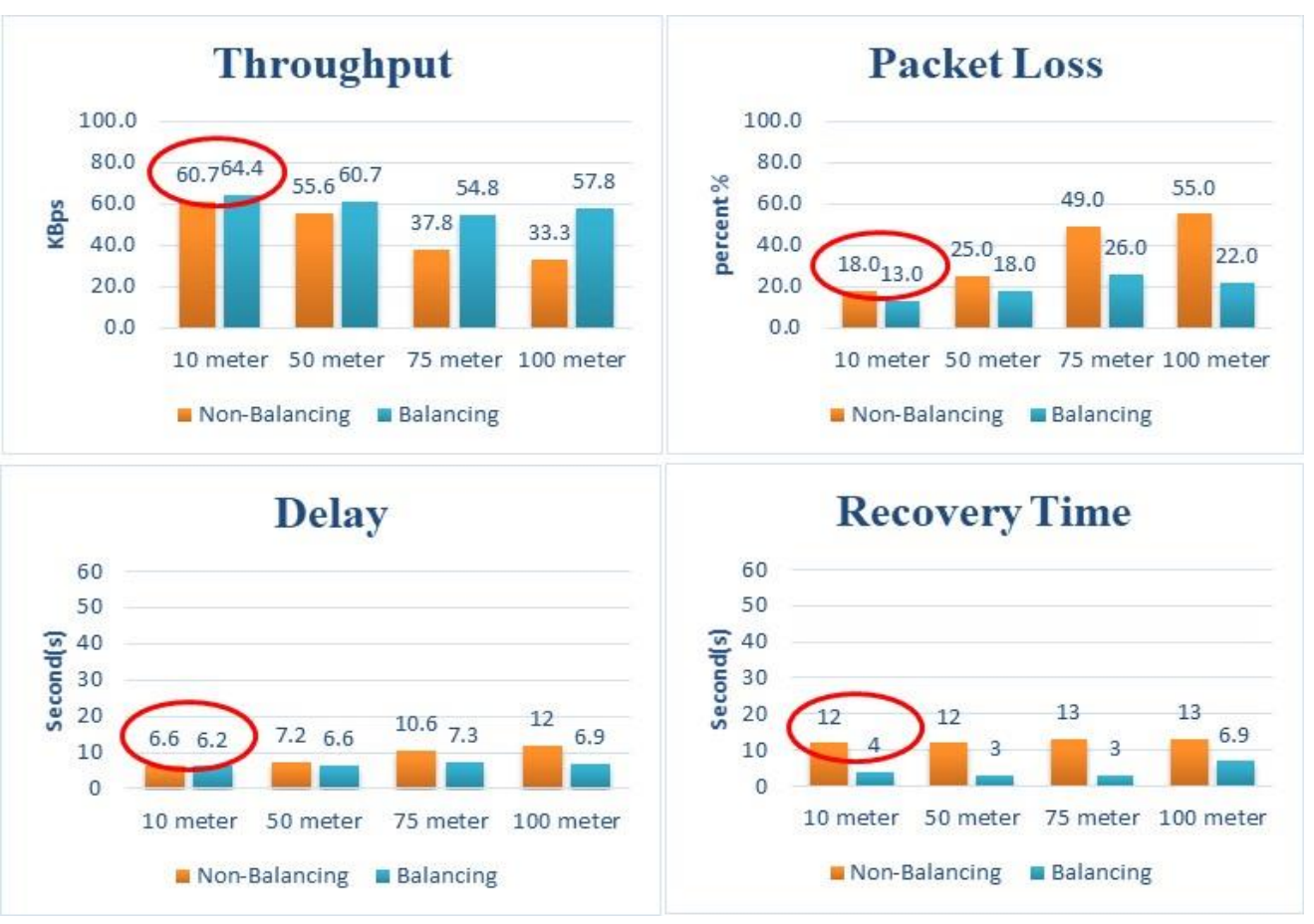

Figure 11. Graphic results of measurement

In measuring packet loss this is also one of the supporting points of the reliability aspect. If the reliability value is relatively small, it represents insignificant network performance. Packet loss analysis shows an average of the non-balancing of $37 \%$ and the balancing of $20 \%$. It means that of the two rule performances above, the rule balancing shows a smaller packet loss compared to the rule balancing. It shows a significant increase in the reliability aspect. It shows a significant increase in the reliability aspect. From this analysis, distance also affects the process of transmitting data packets, the farther the distance between the sender and the receiver, the longer the response and the loss of packet loss performance.

The best criterion of the delay test is to obtain the smallest value of time, the smaller the value, the better the data transmission. Delay analysis shows that the delay of the non-balancing is 9.1 and the balancing is 6.8. It means that of two rule performances, the non-balancing is slower than the balancing.

The fit criterion of this measurement is to obtain the recovery time value ( $\mathrm{rt}$ ) closer to 0 second, the faster the recovery time in fixing problems on damaged routes/errors, the more effective the rule performance. Of the two rules, the rule balancing has better measurement result in terms of recovery when the route is damaged.

Figure 11 indicates that the result of the comparison data consists of two main aspects, the reference for network performance, namely reliability, and effectiveness. Reliability is based on package quantity reference, while effectiveness is based on time reference, whereas RSSI parameter $(-\mathrm{dBm})$ is not displayed in the figure, since it has a different measurement unit. The calculation presented in the form of graph is an average calculation of the two parameters. The result based on Reliability has a difference of $17 \%$, based on packet loss, either $\sum$ PacketReceived or $\sum$ PacketLoss. This calculation is obtained from the formula in Table 5. 
Table 5. Reliability and Effectiveness Calculation

\begin{tabular}{|c|c|}
\hline Average of Reliability & Average of Effectiveness \\
\hline $\begin{array}{l}=\sum \text { throughput balancing }-\sum \text { throughput non balancing } \\
=54.4-46.9=12.6 \mathrm{bps}\end{array}$ & $\begin{array}{l}=\sum \text { delay non balancing }-\sum \text { delay balancing } \\
=9.1-6.8=2.3 \text { second }\end{array}$ \\
\hline
\end{tabular}

It shows that system balancing contributes to the increased reliability. The result based on effectiveness shows that the rule balancing is superior in the parameter of "delay" and "recovery time" compared to the non-balancing rule because when using the non-balancing system rule, it requires an additional time of 12.5 seconds to switch addresses when the router has a problem. However, on the "recovery time" the rule balancing parameter is faster than the non-balancing because the router in the rule balancing does not need to reset the AT command, so that it can shorten packet transmission time.

The difference in Figure 12 can be seen in $\sum$ PacketLoss where the use of rule balancing produces the number of packets more effective than the rule non-balancing that experience a lot of packet loss due to the absence of control on the router. Based on the standard packet loss parameters in QoS, if packet loss occurs up to $>25 \%$, it is categorized as "Poor" [26].

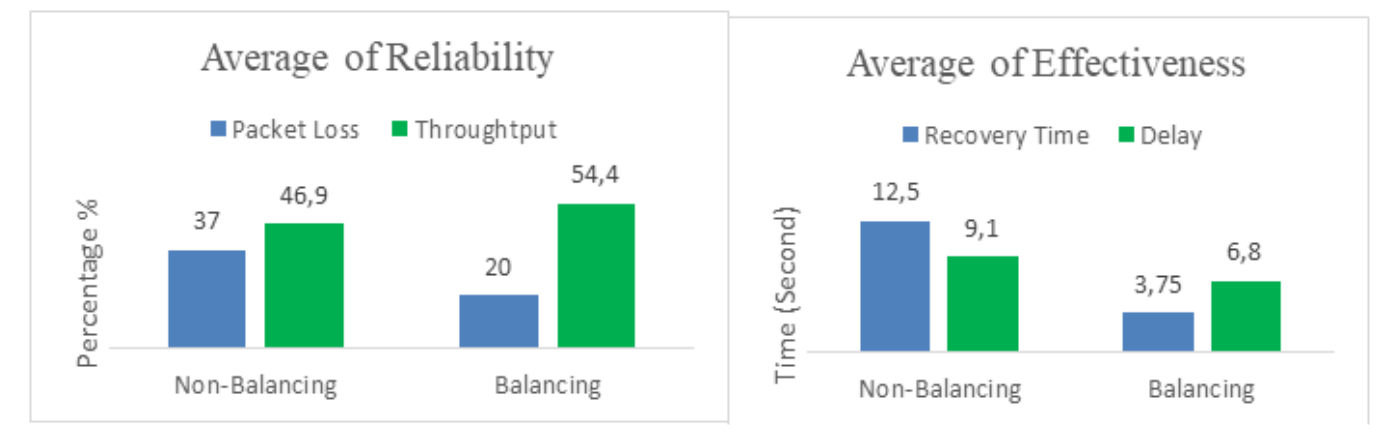

Figure 12. Comparison between the average of reliability and effectiveness

\subsection{Comparison Research}

This section provides an overview of several research focusing on wireless sensor network. We compared three results. This comparison is only a reference in terms of quality from the previous research. Each comparison has four measurement parameters that are used as references for QoS measurements including packet loss, throughput, delay accuracy, and recovery time. The following is the result of the measurement comparison shown in Table 6.

Table 6. Comparison Table of Research

\begin{tabular}{|c|c|c|c|c|}
\hline No & 1. Soijoyo Sigit-2017 [27] & 2. Hafizh Prihtiadi-2017 [28] & 3. Yusuf A-2019 - This Research & 4. Shafaa Laqtib-2019 [29] \\
\hline \multirow[t]{2}{*}{1} & Packet Loss Accuracy & Packet Loss Accuracy & Packet Loss Accuracy & Packet Loss Accuracy \\
\hline & $97-99 \%$ & $91.3-98.8 \%$ & $82-87 \%$ & $64-69 \%$ \\
\hline \multirow[t]{2}{*}{2.} & Troughput Accuracy & Troughput Accuracy & Troughput Accuracy & Troughput Accuracy \\
\hline & $30-32 \mathrm{KBps}$ & - & $60.7-64.4 \mathrm{KBps}$ & $22-56 \mathrm{KBps}$ \\
\hline \multirow[t]{2}{*}{3.} & Delay Accuracy & Delay Accuracy & Delay Accuracy & Delay Accuracy \\
\hline & $0-0.20$ second & - & $6.2-6.6$ second & $5-10$ second \\
\hline \multirow[t]{2}{*}{4.} & Recovery Time Accuracy & Recovery Time Accuracy & Recovery Time Accuracy & Recovery Time Accuracy \\
\hline & - & - & $4-12$ second & - \\
\hline
\end{tabular}

The result of the packet loss comparison indicates that this research remains in the medium category and is in the third position in terms of packet loss because the level of data accuracy of packet delivery is in the range of $85 \%$, in good category [26]. The throughput aspect of this study provides the best measurement 
ranging from 60.7 to $64.4 \mathrm{Kbps}$ among other studies. The expected criteria from the measurement of throughput is the greater throughput value represents the larger bandwidth allocation for transferring data. The aspect of delay comparison shows a slower value compared to the other three results. This is because in system load balancing there is a switching address on the router that gives additional time to data transmission. However, the recovery time of this study generates a new contribution in terms of network wireless sensors using Mesh topologies that had never been measured in the previous research. The expected criterion of this measurement is to obtain recovery time (rt) closer to 0 second, the faster the recovery time in fixing problems on damaged routes/errors, the more effective the rule performance.

\section{CONCLUSION}

The dynamic routing system has proven to be effective in improving the quality of WSN network reliability compared to using static routing that is unable to provide alternative routes when the router faces problems. The impact of a router unable to provide alternative routes when a problem occurs will further trigger packet loss. Therefore, the proposed dynamic routing system is able to reduce packet loss by $17 \%$, where the calculation is supported by two measurement aspects based on packet loss and throughput, which further proves that dynamic routing system has in improving the quality of WSN network reliability.

The application of load balancing system generates increased throughput (capacity), compared to the non-balancing. The non-balancing performance only forwards data packet without any control and selfhealing on the router, therefore, when the router is overloaded, there will not be any response which can reduce the quality of network performance. From the result, it can be seen that the network effectiveness in terms of recovery time aspect increases by 8.75 seconds and the aspect delay of 2.3 seconds.

\section{ACKNOWLEDGMENTS}

The authors give a deep thanks to God Almighty, to Institut Teknologi Sepuluh Nopember, to The Ministry of Research, and Higher Education of Indonesia support this research. We appreciate the supportive comments or suggestions for improving this research.

\section{REFERENCES}

[1] R. Piyare and S. Lee, "Performance analysis of xBee ZB module based wireless sensor networks," Int. J. Sci. Eng. Res., vol. 4, no. 4, pp. 1615-1621, 2013.

[2] J. N. Al-Karaki and A. E. Kamal, "Routing techniques in wireless sensor networks: a survey," IEEE Wirel. Commun., vol. 11, no. 6, pp. 6-27, 2004.

[3] S. V Gharge, P. S. Dharavath, and V. Ghorpade, "Wireless sensor network for smart agriculture," 2nd Int. Conf. Appl. Theor. Comput. Commun. Technol., vol. 3, no. 2, pp. 1377-1380, 2014.

[4] D. Fanfang, Z. Dahai, B. Zhiqian, C. Xin, D. Xinzhou, and S. Shenxing, "ZigBee wireless networking for the interlligent protection center research," Prepr. 5th Int. Conf. Electr. Util. Deregul. Restruct. Power Technol., pp. 7-10, 2015.

[5] Z. Dzulkurnain, A. K. Mahamad, and S. Saon, "Internet of things (IoT) based traffic management \& routing solution for parking space," Indonesian Journal of Electrical Engineering and Computer Science (IJEECS), vol. 15, no. 1, pp. 336-345, 2019.

[6] V. W. Mahyastuty and M. S. Arifianto, "Wireless sensor network over high altitude platform," TELKOMNIKA (Telecommunication, Computing, Electronics and Control), vol. 16, no. 1, pp. 129-133, 2018.

[7] M. Iqbal, M. Fuad, H. Sukoco, and H. Alatas, "Hybrid tree-like mesh topology as new wireless sensor network platform," TELKOMNIKA (Telecommunication, Computing, Electronics and Control), vol. 14, no. 3, pp. 1166-1174, 2016.

[8] S. Navulur, A. S. C. S. Sastry, and M. N. G. Prasad, "Agricultural management through wireless sensors and internet of things," International Journal of Electrical and Computer Engineering (IJECE), vol. 7, no. 6, pp. 3492-3499, 2017.

[9] H. Z. Kotta, K. Rantelobo, S. Tena, and G. Klau, "Wireless sensor network for landslide monitoring in nusa tenggara timur," TELKOMNIKA (Telecommunication, Computing, Electronics and Control), vol. 9, no. 1, pp. 9-18, 2011.

[10] R. Sarno and D. R. Wijaya, "Recent development in electronic nose data processing for beef quality assessment," TELKOMNIKA (Telecommunication, Computing, Electronics and Control), vol. 17, no. 1, 2019.

[11] W. Jung and S. H. Cho, "Load balancing system with sub-network management in wireless sensor networks," Proc. - 2010 2nd IEEE Int. Conf. Netw. Infrastruct. Digit. Content, IC-NIDC 2010, no. September, pp. 639-643, 2010.

[12] S. Shivapur, S. G. Kanakaraddi, and A. K. Chikaraddi, "Load balancing techniques in wireless sensor networks : a comparative study," vol. 14, no. 2, pp. 218-223, 2015.

[13] G. Gautam and B. Sen, "Design and simulation of wireless sensor network topologies using the zigbee standard," Int. J. Comput. Appl., vol. 113, no. 16, pp. 14-16, 2015. 
[14] A. A. M. Khalaf and M. S. A. Mokadem, "Effects of zigbee component failure on the wsn performance with different topologies,” Proc. Int. Conf. Microelectron. ICM, no. December, pp. 9-12, 2017.

[15] O. Yusuf, "Determination of optimal power for zigbee-based wireless sensor networks," University of Windsor Windsor, Canada, 2014.

[16] R. S. Issa, "Study of multiple-source data collection in wireless sensor networks," Indonesian Journal of Electrical Engineering and Computer Science (IJEECS), vol. 14, no. 3, pp. 1479-1483, 2019.

[17] S. M. Chaitanya, "Analysis of power consumption of an end device in a zigbee mesh network," The University of North Carolina at Charlotte, 2007.

[18] Y. Ardiansyah, R. Sarno, and O. Giandi, "Rain detection system for estimate weather level using mamdani fuzzy inference system," in 2018 International Conference on Information and Communications Technology, ICOIACT 2018, 2018, vol. 2018-Januari, pp. 848-854.

[19] F. Robert, Building wireless sensor networks, First Edit. United States of America: O'Reilly Media, Inc, 2010.

[20] H. Kumbhar, "Wireless sensor network using xbee on arduino platform an experimental study," Int. Conf. Comput. Commun. Control Autom., 2016.

[21] D. R. Wijaya, R. Sarno, and E. Zulaika, "Information quality ratio as a novel metric for mother wavelet selection," Chemom. Intell. Lab. Syst., vol. 160, pp. 59-71, 2017.

[22] D. R. Wijaya, R. Sarno, and E. Zulaika, "Sensor array optimization for mobile electronic nose: wavelet transform and filter based feature selection approach," Int. Rev. Comput. Softw., vol. 11, no. 8, pp. 659-671, 2016.

[23] D. R. Wijaya, R. Sarno, and E. Zulaika, "Gas concentration analysis of resistive gas sensor array," 2016 Int. Symp. Electron. Smart Devices, ISESD 2016, pp. 337-342, 2017.

[24] L. Zhang and F. Chen, "A round-robin scheduling algorithm of relay-nodes in wsn based on self-adaptive weighted learning for environment monitoring," J. Comput., vol. 9, no. 4, pp. 830-835, 2014.

[25] K. H. Mahmoud, "Data collection and processing from distributed system of wireless sensors," Masaryk Unviversity, 2013.

[26] W. Sugeng, J. E. Istiyanto, K. Mustofa, and A. Ashari, "The impact of qos changes towards network performance," Int. J. Comput. Networks Commun. Secur., vol. 3, no. 2, pp. 48-53, 2015.

[27] S. Soijoyo and A. Ashari, "Analysis of zigbee data transmission on wireless sensor network topology," Int. J. Adv. Comput. Sci. Appl., vol. 8, no. 9, pp. 145-151, 2017.

[28] H. Prihtiadi and M. Djamal, "The reliability of wireless sensor network on pipeline monitoring system," vol. 49, no. 1, pp. 51-56, 2017.

[29] S. Laqtib, K. El Yassini, M. L. Hasnaoui, and A. Info, "Link-state qos routing protocol under various mobility models," Indonesian Journal of Electrical Engineering and Computer Science (IJEECS), vol. 16, no. 2, pp. 906-916, 2019.

\section{BIOGRAPHIES OF AUTHORS}
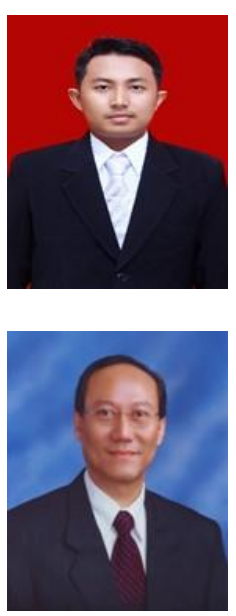

A. Yusuf Ardiansyah is currently a teacher and working toward the Master of Technology Management degree from Institut Teknologi Sepuluh Nopember. He received his bachelor's degree in Computer Engineering from Electronic Engineering Polytechnic Institute of Surabaya (PENS), Surabaya, Indonesia in 2012. He has been working as Teacher in Computer and Network Engineering Department at SMK Rajasa Surabaya, Indonesia. His research interest includes Wireless Sensor Network, Internet of Thing, Network Analyst and Computer Networks. E-mail : yusuf.17092@mhs.its.ac.id, yusufardian.tanz@gmail.com

Riyanarto Sarno is a Professor, Informatics Department, Institut Teknologi Sepuluh Nopember, Surabaya, Indonesia. He received the bachelor's degree in Electrical Engineering from Institut Teknologi Bandung, Bandung, Indonesia in 1987. He received M.Sc and Ph.D. in Computer Science from the University of Brunswick Canada in 1988 and 1992, respectively, in 2003 he was promoted to a Full Professor. His teaching and research interest include Internet of Thing, Process-Aware Information System, Intelligent System, and Smart Grid.

E-mail: riyanarto@if.its.ac.id 